\title{
IFN- $\gamma$, IL-2, IP-10, and MIG as Biomarkers of Exposure to Leishmania spp., and of Cure in Human Visceral Leishmaniasis
}

\author{
Ana V. Ibarra-Meneses ${ }^{1}$, Prakash Ghosh ${ }^{2}$, Faria Hossain ${ }^{2}$, Rajashree Chowdhury ${ }^{2}$, \\ Dinesh Mondal ${ }^{2}$, Jorge Alvar ${ }^{3}$, Javier Moreno ${ }^{1}$ and Eugenia Carrillo ${ }^{1 *}$ \\ ${ }^{1}$ WHO Collaborating Centre for Leishmaniasis, Centro Nacional de Microbiologia, Instituto de Salud Carlos, Madrid, Spain, \\ ${ }^{2}$ Nutrition and Clinical Services Division, International Centre for Diarrhoeal Disease Research, Dhaka, Bangladesh, \\ ${ }^{3}$ Drugs for Neglected Diseases Initiative, Geneva, Switzerland
}

OPEN ACCESS

Edited by:

Brice Rotureau,

Institut Pasteur, France

Reviewed by:

Hira Nakhasi,

Center for Biologics Evaluation and

Research (FDA), United States

Fabienne Tacchini-Cottier,

University of Lausanne, Switzerland

${ }^{*}$ Correspondence:

Eugenia Carrillo

ecarrillo@isciii.es

Received: 27 February 2017 Accepted: 04 May 2017

Published: 31 May 2017

Citation:

Ibarra-Meneses AV, Ghosh P, Hossain F, Chowdhury R, Mondal D, Alvar J, Moreno J and Carrillo E (2017)

IFN- $\gamma$, IL-2, IP-10, and MIG as

Biomarkers of Exposure to Leishmania spp., and of Cure in Human Visceral

Leishmaniasis.

Front. Cell. Infect. Microbiol. 7:200.

doi: 10.3389/fcimb.2017.00200
New biomarkers are needed for monitoring the effectiveness of treatment for visceral leishmaniasis (VL). They might also improve the detection of the asymptomatic population in Leishmania-endemic areas. This paper examines the IL-2, IFN- $\gamma$, IFN- $\gamma$-induced protein 10 (IP-10), and monokine-induced-by-IFN- $\gamma$ (MIG) levels in whole blood-stimulated in vitro with soluble Leishmania antigen (SLA) - taken from asymptomatic individuals and patients treated for VL living in a post-outbreak (Leishmania infantum) area in Spain, and in an endemic (Leishmania donovani) area of Bangladesh. IP-10 was found to be an accurate global marker of asymptomatic subjects with positive cellular/humoral tests, while MIG was found to be a better marker of contact with $L$. donovani than IL-2 but no for those with $L$. infantum. Determining IP-10, MIG, and IFN- $\gamma$ levels proved useful in monitoring the cellular immune response following treatment for active disease caused by $L$. infantum.

Keywords: chemokine, cytokine, biomarker, leishmaniasis, whole blood assay, asymptomatic, treatment

\section{INTRODUCTION}

Visceral leishmaniasis (VL), or kala-azar, is one of the deadliest and most neglected of all tropical diseases. Effective therapy is central to any strategy for controlling leishmaniasis, and biomarkers able to indicate the initiation of a successful response might help shorten the duration of treatment. The identification and management of asymptomatic subjects has become an increasingly important challenge in VL control programs, yet no validated markers of asymptomatic infection exist, and the parasitological, molecular and serological tools currently used are not entirely suitable (Vallur et al., 2016). There is a therefore a pressing need for new biomarkers that can identify the asymptomatic population in areas where Leishmania is endemic, and that can aid in monitoring the success of treatment in patients with active disease.

IFN- $\gamma$ in soluble Leishmania antigen (SLA)-stimulated whole blood (in vitro) has recently been described to gradually but significantly increase after the successful treatment of patients infected with Leishmania donovani (Adem et al., 2016) and Leishmania infantum (IbarraMeneses et al., 2016). However, this assay has never been used to monitor individual patients from the time of their having active disease through to their cure. IFN- $\gamma$ and IL-2 concentrations following whole blood stimulation with SLA have also been shown to provide accurate markers of asymptomatic L. donovani (Gidwani et al., 2011) and 
L. infantum infection (Ibarra-Meneses et al., 2016). However, despite their high sensitivity and sensitivity, IFN- $\gamma$ and IL-2 are only produced in small amounts; more robust biomarkers would be preferable.

Interest has recently grown in the use of chemokines as alternative biomarkers. When activated by IFN- $\gamma$, many cell types produce IFN- $\gamma$-inducible protein-10 (IP-10) and monokineinduced-by-IFN- $\gamma$ (MIG; Gasperini et al., 1999), which further increase the production of IFN- $\gamma$. Both chemokines provide accurate biomarkers of a range of infections, including those of Mycobacterium tuberculosis, hepatitis C virus and malaria parasites (Azzurri et al., 2005; Armah et al., 2007; Falconer et al., 2010). In recent years, chemokines have been identified in the host response against Leishmania (Oghumu et al., 2010). Indeed, IP-10 has been shown critical in the induction of cellular immunity following vaccination against $L$. donovani (Fallahi et al., 2016), and to play a protective role in reducing the number of intracellular parasites in cutaneous lesions (Vasquez and Soong, 2006). Further, plasma MIG and IP-10 have been shown to increase in patients treated for $L$. donovani-induced VL (Hailu et al., 2004). These chemokines might therefore provide a sensitive means of detecting antigen-specific T-cell responses following Leishmania infection.

The present work examines IP-10 and MIG concentrations in SLA-stimulated whole blood as potential markers of asymptomatic individuals with $L$. infantum/L. donovani infection. The usefulness of these chemokines and of the cytokines IFN- $\gamma$ and IL-2 in monitoring the cellular immune response, and therefore as markers of cure in patients treated for VL, was also investigated.

\section{MATERIALS AND METHODS \\ Data Collection and Participants}

Three hundred and five blood donors attending the Hospital de Fuenlabrada Blood Bank, plus 12 patients with active VL and another 14 cured patients (all cared for by the same hospital's Internal Medicine Department), were recruited over 2015-2016. All were aged $\geq 18$ years and resided in the town of Fuenlabrada (previously described as a focus of L. infantum transmission). Blood samples from all the above were subjected to peripheral blood mononuclear cell isolation and to the in vitro cell proliferation assay (CPA), performed as previously described (Ibarra-Meneses et al., 2016). Of the 305 blood bank samples, 57 were CPA-positive (stimulation index $\geq 2.27$ ); the subjects providing these samples were thus considered asymptomatic (AS-Li) and their blood was selected for further analysis of cytokines and chemokines. Fifty CPA-negative samples were randomly selected as negative endemic L. infantum controls (NVL-Li). Six of the 12 patients with active VL, all treated with liposomal amphotericin B (WHO, 2010), underwent medical examination at $0,3,6$, and 12 after the start treatment, at which times their blood was also sampled. Following WHO guidelines, treated patients were considered cured (CVL) if they showed no relapse by 6 months after beginning treatment (WHO, 2010) - a criterion met by all the present treated patients.
In October 2016, 25 healthy subjects, all $\geq 18$ years of age, were also recruited from Mymensingh in Bangladesh, an area endemic for L. donovani-induced VL. Of these subjects, 12 were classified as asymptomatic (AS-Ld) [based on the rK39 and direct agglutination (DAT) tests] and 13 as negative endemic L. donovani controls (NVL-Ld). Table 1 shows the clinical characteristics of the final study subjects.

\section{Ethics Statement}

Recruitment and sample collection was performed in accordance with guidelines for good clinical practice. The study was approved by the Hospital de Fuenlabrada Ethics and Research Committee, and by the Ethical Review Committee of the International Centre for Diarrhoeal Disease Research, Bangladesh. All study subjects provided their informed written consent to be included.

\section{rK39 Immunochromatographic Test}

The assay was performed using the dipstick format Kalazar Detect Rapid test (InBIOS International, Seattle, WA). Antibody detection was performed with plasma samples according to the manufacturer's instructions.

\section{Direct Agglutination Test}

DAT was performed using freeze-dried antigen (ITMA-DAT/VL; Prince Leopold Institute of Tropical Medicine, Antwerp, Belgium) following the manufacturer's recommendations. Serum samples with a titre of 1:3200 were considered positive.

\section{DNA Extraction and Real-Time PCR}

DNA was extracted from $100 \mu \mathrm{l}$ of peripheral blood by conventional phenol-chloroform extraction and eluted in $100 \mu \mathrm{l}$ sterile distilled water, as previously described (Cunha et al., 2013). Real-time PCR (qPCR) was then performed to detect leishmanial DNA using $4 \mu \mathrm{l}$ of the extracted DNA (Cruz et al., 2013) and primers R223 (1,000 nM) and R333 (500 nM; SigmaAldrich; both for the small subunit rRNA gene), employing the LightCycler FastStart DNA Master SYBR Green I kit (Roche Applied Science).

\section{Preparation of Soluble $L$. infantum Antigen for Stimulation of Whole Blood Collected from Subjects}

L. infantum antigen extract was prepared from stationary phase promastigote cultures (JPC strain, MCAN/ES/98/LLM-722) as previously described (Carrillo et al., 2015). Briefly, parasites resuspended in lysis buffer $(50 \mathrm{mM}$ Tris/5 mM EDTA/HCl, $\mathrm{pH}$ 7) were subjected to three rapid freeze/thaw cycles followed by three $20 \mathrm{~s} 40 \mathrm{~W}$ pulses with a sonicator. Two consecutive $27,000 \mathrm{~g}$ centrifugations for $20 \mathrm{~min}$ at $4^{\circ} \mathrm{C}$ were then performed, and the supernatants collected, aliquoted, and stored at $-80^{\circ} \mathrm{C}$ until use. Protein quantification was performed using the Bradford method employing the Bio-Rad Protein Assay kit (Bio-Rad, USA).

\section{Whole Blood Stimulation Assay}

Whole blood samples were stimulated as previously described (Carrillo et al., 2015; Ibarra-Meneses et al., 2016). Briefly, for 
TABLE 1 | Clinical characteristics of the study population.

\begin{tabular}{|c|c|c|c|c|c|c|c|}
\hline Group & No. of subjects & $\begin{array}{l}\text { Subjects age (y) } \\
\quad(\text { mean/SD) }\end{array}$ & $\begin{array}{c}\text { Sex (No. of females/ } \\
\text { males) }\end{array}$ & $\begin{array}{l}\text { No. (\%) CPA- } \\
\text { positive }\end{array}$ & $\begin{array}{c}\text { No. }(\%) \text { qPCR- } \\
\text { positive }\end{array}$ & $\begin{array}{c}\text { No. }(\%) \text { rK39- } \\
\text { positive }\end{array}$ & $\begin{array}{c}\text { No. (\%) DAT- } \\
\text { positive }\end{array}$ \\
\hline CVL & 14 & $45 \pm 10$ & $5 / 9$ & $14(100)$ & $3(21)$ & $7(50)$ & $8(57)$ \\
\hline Active VL & 12 & $45 \pm 12$ & $5 / 7$ & 0 & $12(100)$ & $8(67)$ & $10(83)$ \\
\hline AS-Li & 57 & $42 \pm 6$ & $15 / 42$ & 57 (100) & 0 & 0 & 0 \\
\hline NVL-Li & 50 & $43 \pm 12$ & $15 / 35$ & 0 & 0 & 0 & 0 \\
\hline AS-Ld & 12 & $38 \pm 10$ & $5 / 7$ & nd & 0 & $11(91.7)$ & $12(100)$ \\
\hline NVL-Ld & 13 & $33 \pm 8$ & $3 / 10$ & nd & 0 & 0 & 0 \\
\hline
\end{tabular}

VL, visceral leishmaniasis; CVL, cured of visceral leishmaniasis; $A S-L i$, asymptomatic subject from $L$. infantum area; $A S$ - $L d$, asymptomatic subject from L. donovani area; NVL-Li, negative control from L. infantum area; NVL-Ld, negative control from L. donovani area; CPA, cell proliferation assay; DAT, direct agglutination test; Nd, not determined.

each sample, an aliquot of blood $(500 \mu \mathrm{L})$ was placed on its own in a tube (negative control), and another in a tube containing $10 \mu \mathrm{g} / \mathrm{ml} \mathrm{SLA}$, and both were incubated at $37^{\circ} \mathrm{C}$ for $24 \mathrm{~h}$. After centrifugation at $2,000 \mathrm{~g}$ for $10 \mathrm{~min}$, the supernatants were collected and stored at $-20^{\circ} \mathrm{C}$ for cytokine/chemokine analysis.

\section{Cytometric Quantification of Cytokines/Chemokines}

IP-10, MIG, IL-2, and IFN- $\gamma$ were quantified in $50 \mu \mathrm{l}$ of plasma from SLA-stimulated whole blood using the BD Cytometric Bead Array Human Flex Set (Becton Dickinson Biosciences, USA) following the manufacturer's instructions. Briefly, $50 \mu \mathrm{l}$ of the plasma of each subject was incubated for $1 \mathrm{~h}$ at room temperature with $50 \mu \mathrm{l}$ of capture beads. After incubation, $50 \mu \mathrm{l}$ of the detection antibody was added and mixture placed $2 \mathrm{~h}$ at room temperature. Data were acquired using a FACSCalibur flow cytometer and analyzed using the Flow Cytometric Analysis Program Array (Becton Dickinson Biosciences, USA). Results for each chemokine/cytokine were expressed as the difference between the SLA-stimulated and control plasma concentrations in $\mathrm{pg} / \mathrm{ml}$.

\section{Statistical Analyses}

Cytokine and chemokine concentrations were compared using the Mann-Whitney $U$-test. The cut-off for each analyte was determined by calculating the area under the receiver operating characteristic (ROC) curve (AUC) and the 95\% confidence interval (CI). Significance was set at $p \leq 0.05$. Analyte concentrations before and after treatment were compared using the Wilcoxon paired $t$-test. All calculations were performed using GraphPad Prism 7.0 software (GraphPad Software, USA).

\section{RESULTS}

\section{IP-10 is a Good Marker of Contact with Visceralizing Leishmania Species, and MIG a Good Marker of Contact with $L$. donovani} IP-10, MIG, IL-2, and IFN- $\gamma$ were analyzed in blood (SLAstimulated) taken from subjects exposed to $L$. infantum and $L$. donovani infection (Figure 1). In asymptomatic individuals from the corresponding areas, all three analytes were found in much higher concentrations than in the respective negative controls (Figures 1A-H, respectively). The median IP-10 concentrations of the AS-Li and AS-Ld subjects were similar $(3,303 \mathrm{pg} / \mathrm{ml}$ compared to $3,406 \mathrm{pg} / \mathrm{ml}$, respectively), and only slight differences were observed in the production of MIG (892 pg/ml in AS-Li subjects and $604 \mathrm{pg} / \mathrm{ml}$ in AS-Ld subjects) and IFN$\gamma(60.07 \mathrm{pg} / \mathrm{ml}$ in AS-Li subjects and $89.94 \mathrm{pg} / \mathrm{ml}$ in AS-Ld subjects). However, a large difference was detected in the IL-2 concentration of the AS-Li and AS-Ld subjects (172.2 and 79.76 $\mathrm{pg} / \mathrm{ml}$, respectively).

The asymptomatic subjects from Bangladesh were seropositive for $L$. donovani, while the asymptomatic subjects from Spain were seronegative for L. infantum (Table 1). qPCR returned negative results for all blood samples.

The detection accuracy for each analyte was determined by ROC analyses (Figure 2). The detection performance of IFN- $\gamma$, 52 of 55 subjects were identified as AS-Li while 10 of 12 subjects were found as AS-Ld (Table 2). IL-2 accuracy was 100\% (55/55) and $83.33 \%(10 / 12)$ for AS-Li and AS-Ld, respectively. IP-10 resulted to be a good biomarker to detect asymptomatic subjects from both areas of study. The AUC for IP-10 was 0.9939 for the AS-Li subjects and 0.9872 for the AS-Ld subjects. IP-10 was able to identify 54 of 55 and 11 of 12 subjects who were infected with $L$. infantum or L. donovani, respectively. In addition, MIG turned out to be the best biomarker to identify asymptomatic subjects from $L$. donovani area. The AUC for MIG was 1.000, and correctly identified $100 \%(12 / 12)$ of asymptomatic subjects. All of the AUC-values were $>0.9300$ and significantly different (Table 2).

\section{IFN- $\gamma$, IP-10, and MIG are Potential Markers for Checking the Effectiveness of Treatment in Patients with $L$. infantum- Induced VL}

IP-10, MIG, IL-2, and IFN- $\gamma$ concentrations were determined in blood (after stimulation with SLA) from 14 CVL and 12 active VL subjects from the L. infantum area (Figure 3). The blood of the CVL subjects produced significantly more IP-10, MIG, IL-2, and IFN- $\gamma$ (median IP-10: 2,638 pg/ml; MIG: 1,033 pg/ml; IL-2: 102 $\mathrm{pg} / \mathrm{ml}$; IFN- $\gamma: 380 \mathrm{pg} / \mathrm{ml}$ ) than did that of the active VL subjects (IP-10: $270.1 \mathrm{pg} / \mathrm{ml}$; MIG: $123.1 \mathrm{pg} / \mathrm{ml}$; IL-2: $0.00 \mathrm{pg} / \mathrm{ml}$; IFN- $\gamma$ : $7.72 \mathrm{pg} / \mathrm{ml})$. No IL-2 was detected in the SLA-stimulated blood of the active VL patients. 

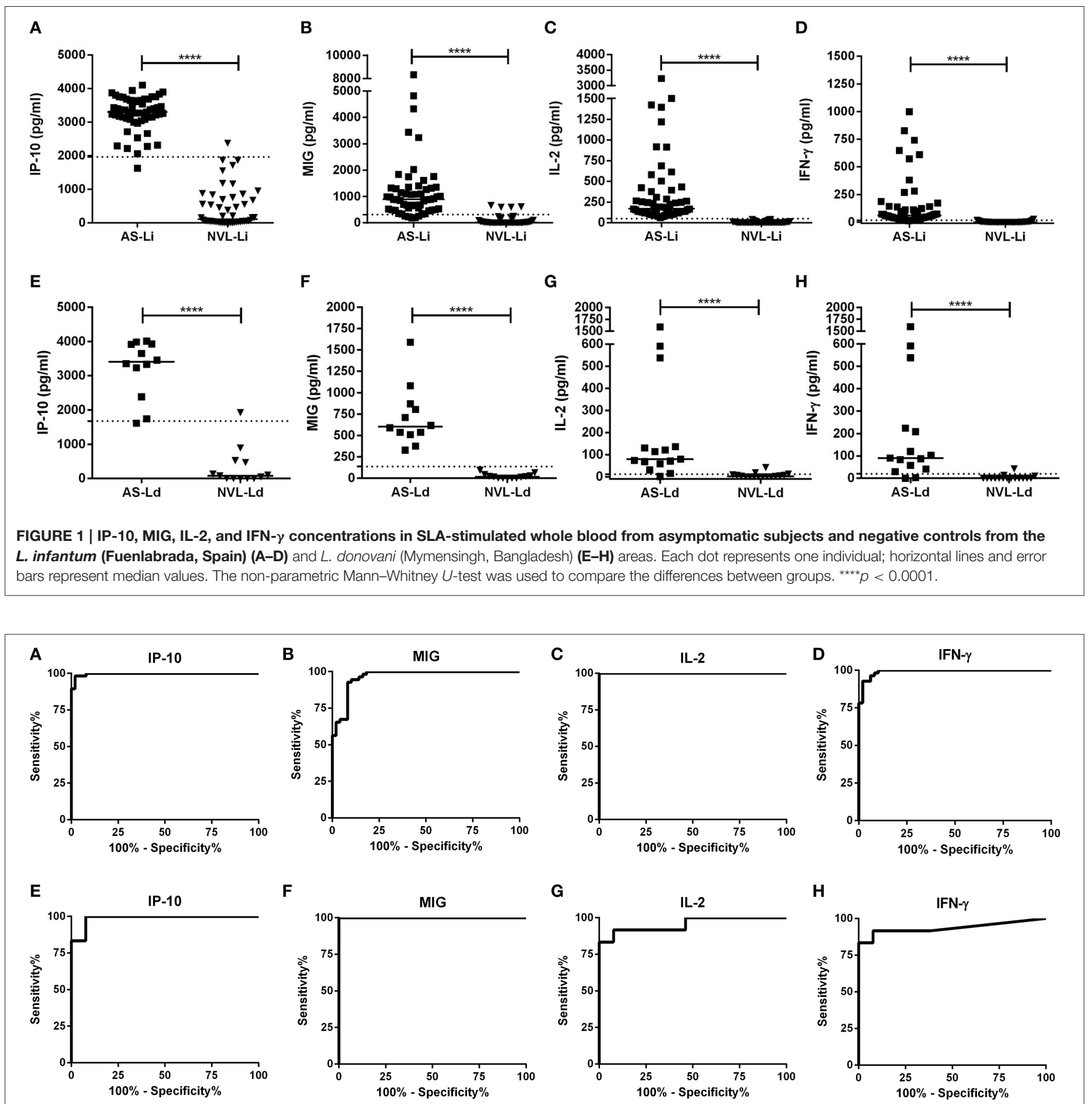

FIGURE 2 | ROC curve analyses for IP-10, MIG, IL-2, and IFN- $\gamma$ in SLA-stimulated whole blood from subjects exposed to $L$. infantum (A-D) and L. donovani (E-H).

At 6 months of follow-up, the AUCs for IP-10, MIG, IL-2, and IFN- $\gamma$ were 0.9881 (95\%CI: 0.9574-1.000), 0.8252 (95\%CI: $0.6360-1.000), 0.9613$ (95\%CI: $0.8767-1.0000)$, and 0.9643 (95\%CI: 0.8909-1.0000), respectively (Figure 3C).

The chemokines/cytokines in the blood (SLA-stimulated) of the six followed subjects with active VL were also quantified over the latters' treatment period. Significant increases in
IP-10, MIG, IL-2, and IFN- $\gamma$ were seen at month 0 compared to non-infected subjects (Table S1). The concentrations of these chemokines/cytokines rose after treatment, with values remaining higher at 12 months (Figure 4A). The highest IP-10 (maximum mean 2,368 pg/ml; $P=0.0026$ ) and MIG (maximum mean $1,384 \mathrm{pg} / \mathrm{ml} ; p=0.0639)$ concentrations were detected during the first months of follow-up (Figure 4A). Increases in 
TABLE 2 | Detection accuracy from asymptomatic subjects by using WBA.

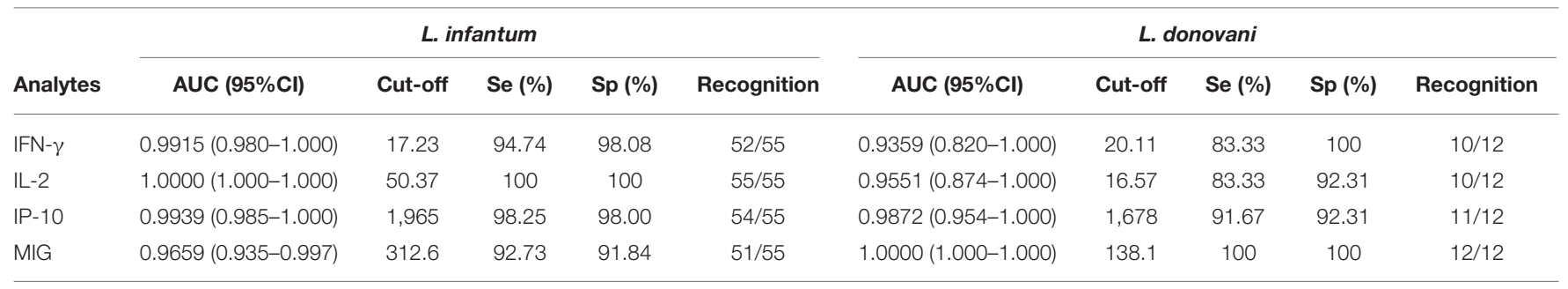

AUC, Area under the curve; Se, sensitivity; Sp, specificity.
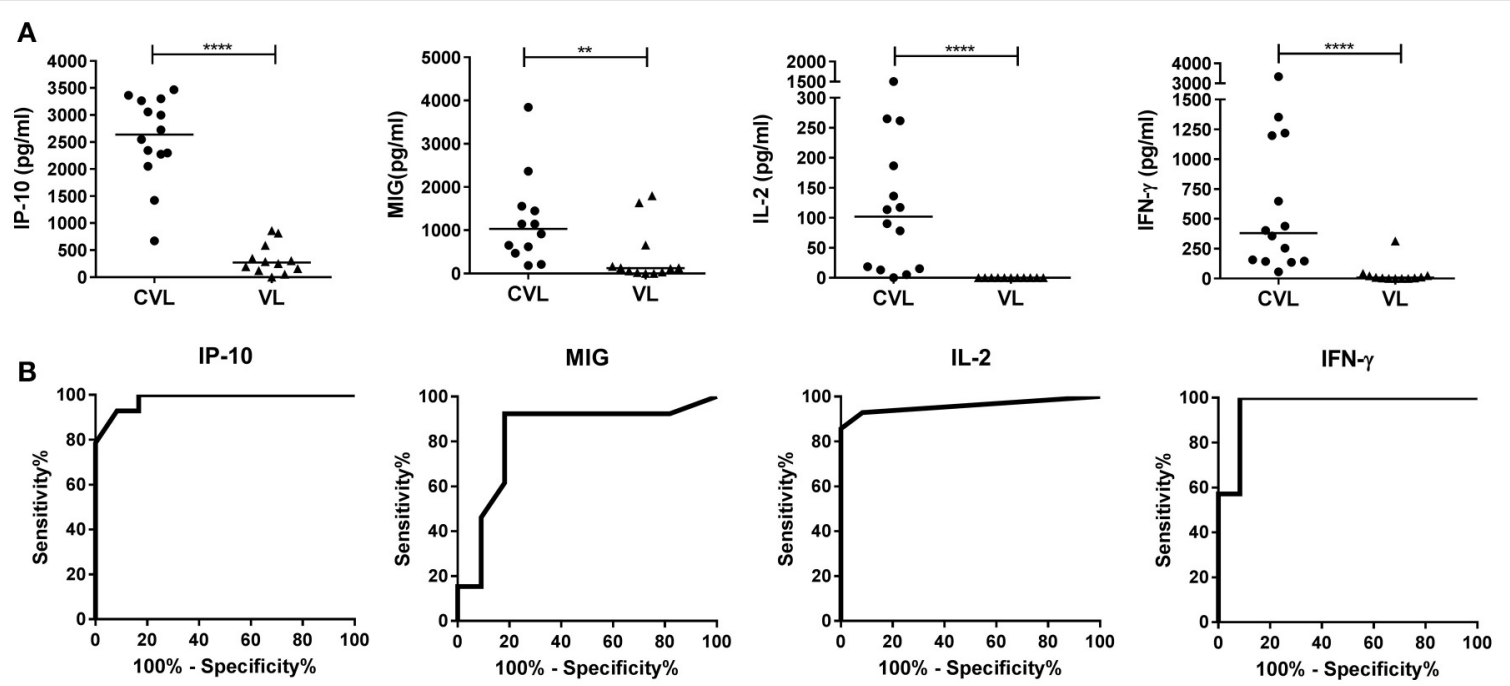

C

\begin{tabular}{lccccc}
\hline Analytes & AUC $(\mathbf{9 5} \% \mathbf{C I})$ & $\boldsymbol{P}$-values & Sensitivity $(\mathbf{9 5} \% \mathbf{C I})$ & Specificity (95\% CI) & Cut-off (pg/ml) \\
\hline IP-10 & $0.9881(0.9574-1.0000)$ & $<0.0001$ & $92.86(61.52-99.79)$ & $100(63.06-100)$ \\
MIG & $0.8252(0.6360-1.0000)$ & 0.0071 & $92.31(63.87-99.81)$ & $81.82(48.22-97.72)$ \\
IL-2 & $0.9613(0.8767-1.0000)$ & $<0.0001$ & $78.57(49.20-95.34)$ & $100(73.54-100)$ & 176 \\
IFN- $\gamma$ & $0.9643(0.8909-1.0000)$ & $<0.0001$ & $91.67(61.52-99.79)$ & $100(61.52-99.79)$ & 14.10 \\
\hline
\end{tabular}

FIGURE 3 | IP-10, MIG, IL-2, and IFN- $\gamma$ concentrations in whole blood (after stimulation with SLA) from CVL and active VL patients from the L. infantum area. Whole blood from $14 \mathrm{CVL}$ and 12 active VL subjects was stimulated for $24 \mathrm{~h}$ with SLA. Analyte concentrations (pg/ml) were obtained by subtracting the values for the negative control plasma from the values for the SLA-stimulated plasma. (A) The horizontal line for the individual biomarkers indicates the median value. (B) ROC curve analyses for IP-10, MIG, IL-2, and IFN- $\gamma$ in blood (SLA-stimulated) from CVL subjects ( $n=14$ ) at 6 month of follow-up. (C) AUC, sensitivity, and specificity values. ${ }^{\star \star} P<0.01,{ }^{\star \star \star \star} P<0.0001$.

IL-2 and IFN- $\gamma$ were also seen (maximum mean $71.2 \mathrm{pg} / \mathrm{ml}, p=$ 0.0108 and $919.5 \mathrm{pg} / \mathrm{ml} ; p=0.0105$, respectively), but were slower compared to those seen for the chemokines studied (Figure 4A). The calculated cut-offs (Figure 3C) showed SLA-induced IFN- $\gamma$ and MIG to distinguish between cured status and active disease status at 6 months in $6 / 6$ patients (100\%); IP-10 distinguished in $5 / 6$ patients, and IL-2 did so in 4/6 (Figure 4B).

\section{DISCUSSION}

Parasitological, molecular, and serological diagnostic tools are used to detect asymptomatic subjects in Leishmania-endemic areas. However, in the present study, all the asymptomatic subjects were qPCR-negative, and while the rK39/DAT test was able to detect asymptomatic subjects infected with $L$. donovani, it could not detect those infected with L. infantum. The combination of these tests with an assay to detect cell-mediated immunity against Leishmania might be the best option when trying to determine the real prevalence of asymptomatic status (Gadisa et al., 2012), and in fact the asymptomatic subjects from the L. infantum area were all found to be CPA-positive. However, the CPA test is laborious and difficult to run in the fieldwhich is why it was not performed in the L. donovani area. The SLA-stimulated whole blood assay, when used in the detection of the new markers identified in the present work, would appear to offer a promising alternative for detecting asymptomatic subjects 
in both L. infantum- and L. donovani-affected areas-including in the field.

We previously reported IL-2 to be the most sensitive and specific biomarker for asymptomatic subjects in $L$. infantum areas (Ibarra-Meneses et al., 2016). Although the present results also show IL-2 to be valuable in this respect, it was not as useful for detecting asymptomatic subjects in the present L. donovani area; indeed, MIG and IP-10 performed much better. Differences found in the IL-2 levels between AS-Li and AS-Ld might be related with a remote or recent infection, respectively, as described in tuberculosis (Krummel et al., 2010). Longitudinal studies with larger numbers of asymptomatic subjects from different endemic areas and characteristic of the cohorts will be needed for a further exploration and clarification of this or other hypothesis. MIG also showed differences as a marker to identify asymptomatic individuals from both endemic areas, being 100\% sensitive and specific for those infected with L. donovani. The high sensitivity and specificity of IP-10 show it can be used as a biomarker for identifying asymptomatic individuals living in both $L$. donovani and $L$. infantum areas. IP-10 and MIG have both been described as potential biomarkers for assessing latent tuberculosis (Lighter et al., 2009; Rubbo et al., 2012), but this is the first study of these chemokines as biomarkers for use with SLA-stimulated blood.

IFN- $\gamma$ in SLA-stimulated whole blood has previously been suggested as a biomarker for identifying patients cured of leishmaniasis. Adem et al. (2016) reported antigen-specific IFN$\gamma$ to be gradually but significantly increased at 3 and 6 months after the start of successful VL treatment in Ethiopia (Adem et al., 2016). In a previous study on patients with L. infantuminduced VL in Spain, we described results comparable to those recorded by Adem et al. for IFN- $\gamma$ and IL- 2 at 3 months after the start of treatment (Ibarra-Meneses et al., 2016). However, Singh et al. (2012) reported that IFN- $\gamma$ levels in SLA-stimulated blood were similar in Indian patients with active VL and in cured patients at least 6 months after successful treatment (Singh et al., 2012). We hypothesize that one of the factors that could be influencing these results is the time when whole blood assay was performed after cure. Present results and others (Adem et al., 2016; Ibarra-Meneses et al., 2016) found that IFN- $\gamma$ increased at 3 and 6 months after start of the treatment, but we described here that mean levels of IFN- $\gamma$ at 12 months experimented a three-fold decrease regarding to 6 months. In the report from India where IFN- $\gamma$ levels were similar in active and cured subjects, whole blood assay was performed at least 6 months after successful treatment but the time is not further specified (Singh et al., 2012). Due to small size of our cohort, further investigation into the influence of the time after cure to perform whole blood assay for the monitoring of immunity is needed. However, other epidemiological factors of the endemic area and nutritional condition of the subjects together with the treatment received may be influencing these results.

The present work is the first to use a whole blood assay to monitor the same individuals from the time of active disease through to their cure, and to study the immune response over

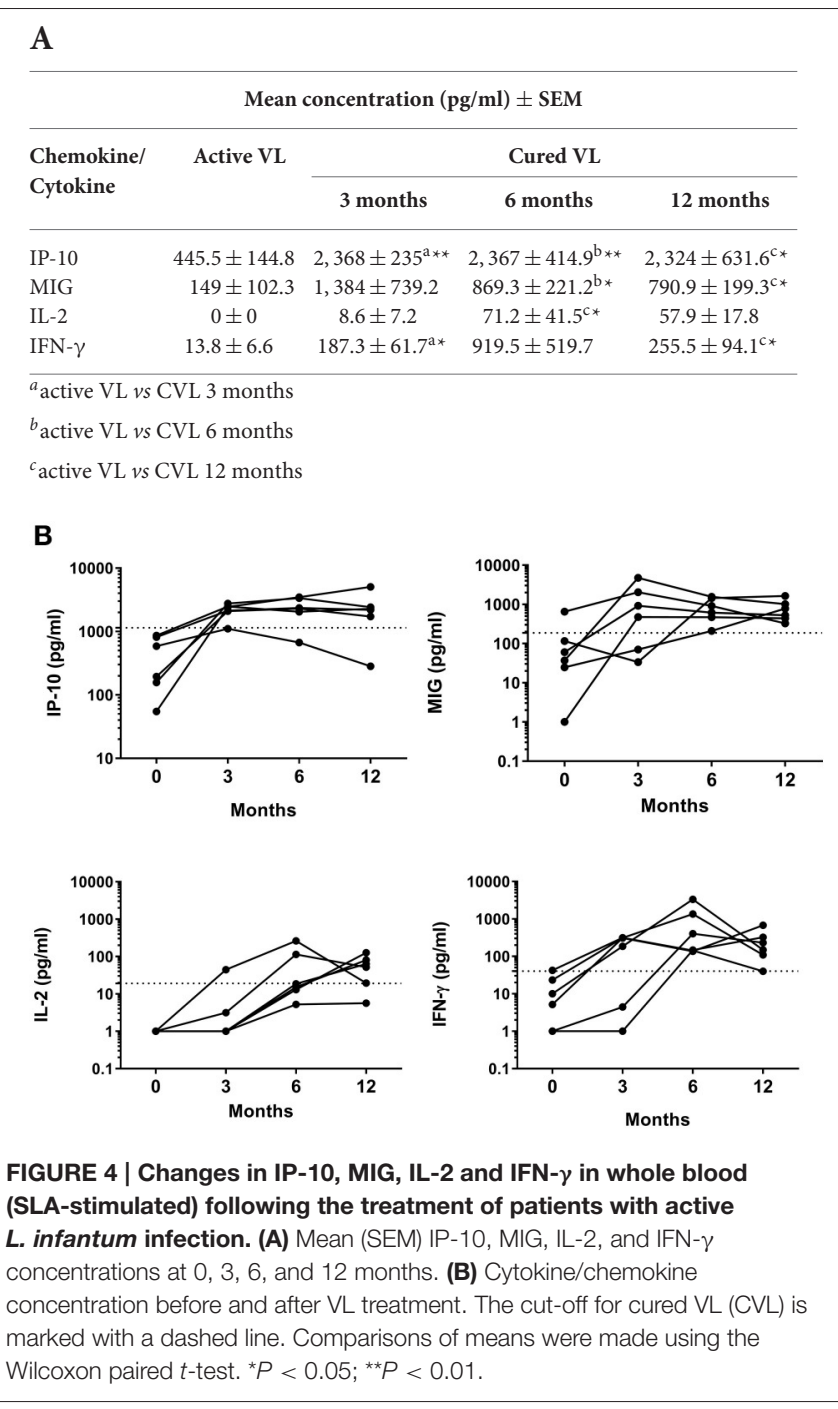

a period of 1 year. The results show IFN- $\gamma$, but not IL-2, to be very useful for monitoring the effectiveness of treatment for VL with liposomal amphotericin B. The results also reveal IP10 and MIG to be excellent markers of cure in patients with $L$. infantum infection. A single previous report records MIG and IP10 as biomarkers of cure in non-SLA stimulated plasma; IFN- $\gamma$, IL-12p40, IL-18, IL-15, IP-10, and MIG concentrations were all markedly elevated in patients with active VL compared to healthy controls (Hailu et al., 2004). IP-10 and MIG are also reported to be markers of recovery from tuberculosis (Azzurri et al., 2005; de Steenwinkel et al., 2012). The present tests should be repeated in immunocompetent individuals from other endemic areas before the accuracy of these chemokines as a marker of "cured" status can be fully established. In addition, studies including subjects treated with other drugs than amphotericin $\mathrm{B}$ are needed before concluding the global use of these markers to identify patient cure. Assessments should also be made in the pediatric population. Cell-mediated immunity tests have recently been proposed for the follow-up of patients co-infected with HIV/Leishmania (Castro et al., 2016). Since IP-10 identifies 
HIV/tuberculosis-infected patients, despite their low CD4 counts (Azzurri et al., 2005; Kassa et al., 2016), it might be used to monitor for relapses of leishmaniasis in HIV/Leishmaniainfected patients.

In conclusion, the present work shows that IP-10 and MIG concentrations can be used to identify asymptomatic subjects infected with $L$. infantum or $L$. donovani. Further, IFN- $\gamma$, MIG and IP-10 can be used as markers of cure in immunocompetent patients treated for $L$. infantum-induced VL. The higher concentrations of IP-10 and MIG than IFN- $\gamma$ and IL- 2 might be easy to detect with simple-to-use platforms (such as ELISAand immunochromatographic-based assays) that could be employed in the field. This minimally invasive, non-sensitizing, simple assay could be of great value in epidemiological studies performed in the field. The better identification of asymptomatic and cured subjects would help in the control of VL. Our group is currently validating the use of IFN- $\gamma$, IL2 , and IP-10 as biomarkers for quantifying the prevalence of asymptomatic status in a cross-sectional study in Madrid (Spain). A study to assess the potential of IFN- $\gamma$, IP-10, and MIG as biomarkers in the follow-up of treated patients is underway in India.

\section{SUMMARY}

This paper reports IP-10 and MIG in SLA-stimulated whole blood to be potential markers of subjects asymptomatic for $L$. infantum and $L$. donovani infection. It also establishes proof of concept that plasma IP-10 may be used as a marker of treatment response and cure in L. infantum-induced visceral leishmaniasis. The very large area under the receiver operating characteristic curves for IP-10 and MIG provides good evidence of the latters' ability to identify asymptomatic subjects and to act as markers of the cellular immune response after treatment. These chemokines appear in much greater concentration than IFN- $\gamma$, and are therefore more robust markers-as described for them regarding the diagnosis of tuberculosis. Testing for multiple biomarkers might allow the results provided by the main marker to be confirmed.

\section{REFERENCES}

Adem, E., Tajebe, F., Getahun, M., Kiflie, A., Diro, E., Hailu, A., et al. (2016). Successful treatment of human visceral leishmaniasis restores antigen-specific IFN-gamma, but not IL-10 production. PLoS Negl. Trop. Dis. 10:e0004468. doi: 10.1371/journal.pntd.0004468

Armah, H. B., Wilson, N. O., Sarfo, B. Y., Powell, M. D., Bond, V. C., Anderson, W., et al. (2007). Cerebrospinal fluid and serum biomarkers of cerebral malaria mortality in Ghanaian children. Malar. J. 6:147. doi: 10.1186/1475-28756-147

Azzurri, A., Sow, O. Y., Amedei, A., Bah, B., Diallo, S., Peri, G., et al. (2005). IFN-gamma-inducible protein 10 and pentraxin 3 plasma levels are tools for monitoring inflammation and disease activity in Mycobacterium tuberculosis infection. Microbes Infect. 7, 1-8. doi: 10.1016/j.micinf.2004. 09.004

Carrillo, E., Carrasco-Anton, N., Lopez-Medrano, F., Salto, E., Fernandez, L., San Martin, J., et al. (2015). Cytokine release assays as tests for

\section{AUTHOR CONTRIBUTIONS}

AI, JA, JM, and EC designed the study; AI, PG, FH, and RC performed experiments; AI analyzed data; AI and EC wrote the manuscript; AI, DM, JA, JM, and EC interpreted the data.

\section{FUNDING}

We gratefully acknowledge the financial support of the Drugs for Neglected Diseases Initiative (DNDi), in turn funded by the Department for International Development (DFID), UK, and the Swiss Agency for Development and Cooperation (SDC), Switzerland. The authors also thank the Instituto de Salud Carlos III which provided funding via the Tropical Diseases Research Network (RICET RD12/0018/0003 and RD12/0018/0008) (www.ricet.es) and the ISCIII-AES project "Impact of a leishmaniasis outbreak in the southwest of Madrid in the immunosuppressed population" (PI13/00440). EC was supported by a research contract funded via the VII PN I+D+I 2013-2016 programme and FEDER Funds (RICET RD12/0018/0003).

\section{ACKNOWLEDGMENTS}

We are very much grateful to the study participants. We thank the staff of the Blood Bank and Haemotherapy Department of the Hospital Universitario de Fuenlabrada and the staff in the SKKRC, for their assistance in the collection of blood samples and to the field staff for detection of asymptomatic cases. We acknowledge the expert advice of Ricardo Molina and Vishal Goyal. We are thankful to icddr,b and its core donor (Government of Bangladesh, UKAid, USAID and Swedish CIDA). We express our gratitude to DNDi for financial support of the study in icddr,b.

\section{SUPPLEMENTARY MATERIAL}

The Supplementary Material for this article can be found online at: http://journal.frontiersin.org/article/10.3389/fcimb. 2017.00200/full\#supplementary-material

exposure to Leishmania, and for confirming cure from Leishmaniasis, in solid organ transplant recipients. PLoS Negl. Trop. Dis. 9:e0004179. doi: 10.1371/journal.pntd.0004179

Castro, A., Carrillo, E., San Martin, J. V., Botana, L., Molina, L., Matia, L., et al. (2016). Lymphoproliferative response after stimulation with Soluble Leishmania Antigen (SLA) as a predictor of visceral leishmaniasis (VL) relapse in HIV+ patients. Acta Trop. 164, 345-351. doi: 10.1016/j.actatropica.2016.09.026

Cruz, I., Millet, A., Carrillo, E., Chenik, M., Salotra, P., Verma, S., et al. (2013). An approach for interlaboratory comparison of conventional and real-time PCR assays for diagnosis of human leishmaniasis. Exp. Parasitol. 134, 281-289. doi: 10.1016/j.exppara.2013.03.026

Cunha, J., Carrillo, E., Sanchez, C., Cruz, I., Moreno, J., and Cordeiro-daSilva, A. (2013). Characterization of the biology and infectivity of Leishmania infantum viscerotropic and dermotropic strains isolated from HIV+ and HIVpatients in the murine model of visceral leishmaniasis. Parasit. Vectors 6:122. doi: 10.1186/1756-3305-6-122 
de Steenwinkel, J. E., de Knegt, G. J., ten Kate, M. T., Verbrugh, H. A., Ottenhoff, T. H., and Bakker-Woudenberg, I. A. (2012). Dynamics of interferon-gamma release assay and cytokine profiles in blood and respiratory tract specimens from mice with tuberculosis and the effect of therapy. Eur. J. Clin. Microbiol. Infect. Dis. 31, 1195-1201. doi: 10.1007/s10096-011-1428-2

Falconer, K., Askarieh, G., Weis, N., Hellstrand, K., Alaeus, A., and Lagging, M. (2010). IP-10 predicts the first phase decline of HCV RNA and overall viral response to therapy in patients co-infected with chronic hepatitis C virus infection and HIV. Scand. J. Infect. Dis. 42, 896-901. doi: $10.3109 / 00365548.2010 .498019$

Fallahi, P., Elia, G., and Bonatti, A. (2016). Leishmaniasis and IFN-gamma dependent chemokines. Clin. Ter. 167, e117-e122. doi: 10.7417/CT.201 6.1954

Gadisa, E., Custodio, E., Canavate, C., Sordo, L., Abebe, Z., Nieto, J., et al. (2012). Usefulness of the rK39-immunochromatographic test, direct agglutination test, and leishmanin skin test for detecting asymptomatic Leishmania infection in children in a new visceral leishmaniasis focus in Amhara State, Ethiopia. Am. J. Trop. Med. Hyg. 86, 792-798. doi: 10.4269/ajtmh.2012.11-0196

Gasperini, S., Marchi, M., Calzetti, F., Laudanna, C., Vicentini, L., Olsen, H., et al. (1999). Gene expression and production of the monokine induced by IFN-gamma (MIG), IFN-inducible T cell alpha chemoattractant (I-TAC), and IFN-gamma-inducible protein-10 (IP-10) chemokines by human neutrophils. J. Immunol. 162, 4928-4937.

Gidwani, K., Jones, S., Kumar, R., Boelaert, M., and Sundar, S. (2011). Interferongamma release assay (modified QuantiFERON) as a potential marker of infection for Leishmania donovani, a proof of concept study. PLoS Negl. Trop. Dis. 5:e1042. doi: 10.1371/journal.pntd.0001042

Hailu, A., van der Poll, T., Berhe, N., and Kager, P. A. (2004). Elevated plasma levels of interferon (IFN)- $\gamma$, IFN- $\gamma$ inducing cytokines, and IFN- $\gamma$ inducible CXC chemokines in visceral leishmaniasis. Am. J. Trop. Med. Hyg. 71, 561-567. doi: 10.4269/ajtmh.2004.71.561

Ibarra-Meneses, A. V., Carrillo, E., Sanchez, C., Garcia-Martinez, J., Lopez Lacomba, D., San Martin, J. V., et al. (2016). Interleukin-2 as a marker for detecting asymptomatic individuals in areas where Leishmania infantum is endemic. Clin. Microbiol. Infect. 22, e1-e4. doi: 10.1016/j.cmi.2016.05.021

Kassa, D., de Jager, W., Gebremichael, G., Alemayehu, Y., Ran, L., Fransen, J., et al. (2016). The effect of HIV coinfection, HAART and TB treatment on cytokine/chemokine responses to Mycobacterium tuberculosis (Mtb) antigens in active TB patients and latently Mtb infected individuals. Tuberculosis 96, 131-140. doi: 10.1016/j.tube.2015.05.015
Krummel, B., Strassburg, A., Ernst, M., Reiling, N., Eker, B., Rath, H., et al. (2010). Potential role for IL-2 ELISpot in differentiating recent and remote infection in tuberculosis contact tracing. PLOS ONE 5:e11670. doi: 10.1371/journal.pone.0011670

Lighter, J., Rigaud, M., Huie, M., Peng, C. H., and Pollack, H. (2009). Chemokine IP-10: an adjunct marker for latent tuberculosis infection in children. Int. J. Tuberc. Lung Dis. 13, 731-736.

Oghumu, S., Lezama-Davila, C. M., Isaac-Marquez, A. P., and Satoskar, A. R. (2010). Role of chemokines in regulation of immunity against leishmaniasis. Exp. Parasitol. 126, 389-396. doi: 10.1016/j.exppara.2010.02.010

Rubbo, P. A., Nagot, N., Le Moing, V., Brabet, M., Bourdin, A., Nogue, E., et al. (2012). Multicytokine detection improves latent tuberculosis diagnosis in health care workers. J. Clin. Microbiol. 50, 1711-1717. doi: 10.1128/JCM.00117-12

Singh, O. P., Gidwani, K., Kumar, R., Nylen, S., Jones, S. L., Boelaert, M., et al. (2012). Reassessment of immune correlates in human visceral leishmaniasis as defined by cytokine release in whole blood. Clin. Vaccine Immunol. 19, 961-966. doi: 10.1128/CVI.00143-12

Vallur, A. C., Reinhart, C., Mohamath, R., Goto, Y., Ghosh, P., Mondal, D., et al. (2016). Accurate serodetection of asymptomatic Leishmania donovani infection by use of defined antigens. J. Clin. Microbiol. 54, 1025-1030. doi: 10.1128/JCM.02620-15

Vasquez, R. E., and Soong, L. (2006). CXCL10/gamma interferon-inducible protein 10-mediated protection against Leishmania amazonensis infection in mice. Infect. Immun. 74, 6769-6777. doi: 10.1128/IAI.01073-06

WHO (2010). Control of the Leishmaniases: Report of a Meeting of the WHO Expert Committee on the Control of Leishmaniases. WHO Technical Report Series.

Conflict of Interest Statement: The authors declare that the research was conducted in the absence of any commercial or financial relationships that could be construed as a potential conflict of interest.

Copyright (c) 2017 Ibarra-Meneses, Ghosh, Hossain, Chowdhury, Mondal, Alvar, Moreno and Carrillo. This is an open-access article distributed under the terms of the Creative Commons Attribution License (CC BY). The use, distribution or reproduction in other forums is permitted, provided the original author(s) or licensor are credited and that the original publication in this journal is cited, in accordance with accepted academic practice. No use, distribution or reproduction is permitted which does not comply with these terms. 